\title{
sciendo
}

\author{
BULGARIAN ACADEMY OF SCIENCES
}

CYBERNETICS AND INFORMATION TECHNOLOGIES • Volume 20, No 6

Special Issue on New Developments in Scalable Computing

Sofia $\bullet 2020$

Print ISSN: 1311-9702; Online ISSN: 1314-4081

DOI: $10.2478 /$ cait-2020-0071

\section{AllerScreener - A Server for Allergenicity and Cross-Reactivity Prediction}

\section{Ivan Dimitrov, Mariana Atanasova}

Faculty of Pharmacy, Medical University of Sofia, 1000 Sofia, Bulgaria

E-mails: idimitrov@pharmfac.mu-sofia.bg matanasova@pharmfac.mu-sofia.bg

\begin{abstract}
Allergenicity of proteins is a subtle property encoded in their structures. The prediction of allergenicity of novel proteins saves time and resources for subsequent experimental work. In the host antigen-presenting cells, the allergens are processed as antigens by the means of Human Leukocyte Antigens (HLA) class II proteins. Sometimes, people allergic to a given protein show allergic reaction to a different protein, even when the two proteins have different routes of exposure. This phenomenon is termed cross-reactivity. Here, we describe a server for allergenicity and cross-reactivity prediction based on the abilities of allergenic proteins to generate binders to HLA class II proteins. The generated peptides are compared to HLA binders originating from known allergens. As a result, the server returns a list of common binders, origin proteins, and species. Different species generate common HLA binders and this determines their crossreactivity. The server is named AllerScreener and is freely accessible at: http//www.ddg-pharmfac.net/AllerScreener.
\end{abstract}

Keywords: AllerScreener, allergenicity, cross-reactivity, EpiTOP, EpiDOCK, HLA class II.

\section{Introduction}

Allergy is abnormal reaction (hypersensitivity) of the immune system to typically harmless substances in the environment [1]. Usually, allergens are soluble protein components of larger particles such as pet dander or tree pollen [2].

The hypersensitivity is developing in two stages: the sensitization stage and the effector (elicitation) stage [2]. In the sensitization stage allergens are recognized as antigens by human $\mathrm{T}$ cells and $\mathrm{B}$ cells. Allergen-specific $\mathrm{T}$ cells drive allergenspecific B cells to produce Immunoglobulin E (IgE). The specific $\operatorname{IgE}$ binds to the surface of mast cells, basophils, and activates eosinophils. In the effector stage upon subsequent re-exposure to the same allergen, the molecules of IgE on the cell surface cross-link the allergen and thus activate the cells and induce inflammatory reactions. 
As a part of the immune mechanisms, binding of antigens to the Major Histocompatibility Complex (MHC) class II proteins is a crucial step in the process of sensitization. It takes place in both antigen recognition by $\mathrm{T}$ cells and activation of $\mathrm{B}$ cells. While the antigen evokes the immune system response, the T cells and B cells do not bind to the entire protein, but only to a peptide fragment called epitope. Not all of the peptides that bind to MHC class II proteins become epitopes, but all of the epitopes are MHC class II binders. In humans, the MHC class II protein complex is encoded by the Human Leukocyte Antigen (HLA) gene complex. There are 3 major MHC class II loci encoded by HLA: HLA-DP, HLA-DQ, and HLA-DR. Human MHC genes are highly polymorphic, i.e. each locus has many alleles. Due to their polymorphism, HLA proteins bind to a large repertoire of antigen peptides. The structure of the binding cleft on the HLA class II proteins limits the length of the binding peptide core to nine residues. Hence, in order to be recognized as an allergen by the immune system, the sequence of a given protein should contain at least one nonamer ( 9 amino acid long peptide) that binds to a HLA class II protein.

The receptor, which is responsible for recognition of T cells is HLA restricted. In general, HLA-DR seems to be the most prevalent restrictive molecule for immunogenic peptides [3]. For allergens, restriction by HLA-DRB1, HLA-DRB3, HLA-DP, and HLA-DQ has been demonstrated. Some T-cell epitopes are restricted by some exclusive HLA class II molecules, but many have been found to be promiscuous and can be presented by diverse HLA class II molecules [4].

At the population level, HLA associations with allergy have been mostly investigated in relation to IgE responses. A lot of evidences for association of hypersensitivity to different type of allergens and specific HLA alleles exist. Studies on allergic rhinitis and asthma have discovered associations with different HLA-DRB1, HLA-DP, and HLA-DQ alleles [5-17]. Studies on allergic bronchopulmonary aspergillosis have found associations only with specific HLA-DRB1 alleles [18-21]. However, in all of them DQB1*02:01 has been found to be a protective marker. Three studies have found associations between specific HLA-DQB1 and HLA-DRB1 alleles and peanut allergy [22-24].

Genome Wide Association Studies (GWAS) allow discovery of associations between different genes and allergic-driven diseases. Associations have been found between allergic asthma [25, 26], allergic rhinitis [27], grass sensitization [28], atopic dermatitis [29] and specific HLA-DRB1, HLA-DP, and HLA-DQ alleles. Another genetic study has identified HLA-DQB1 as the only HLA allele associated with allergic sensitization [30]. Associations have been described between peanut allergy and HLA-DQB1 and HLA-DQA2 [31], between shrimp allergy and HLA-DRB $1 * 04: 05$ and HLA-DQB $1 * 04: 01$, and between peach allergy and HLA-DRB $1 * 09: 01$ and HLA-DQB $1 * 03: 03$ [32].

The sensitization of the human immune system to a specific allergenic protein from a specific species is often related to a reaction to proteins from different species, even with different routes of exposure. This phenomenon is due to cross-reactivity between proteins of different origin. Cross-reactivity between antigens occurs when an antigen-specific antibody binds to a different antigen. The two antigens have similar three-dimensional structural regions, known as conformational epitopes, 
which allow the antibody to be recognized by both antigens $[33,34]$. Some studies have found a strong connection between the reaction of the immune system to allergens and T-cell epitope recognition [35, 36]. The involvement of HLA class II proteins in both T-cell epitope recognition and B-cell activation could be a key for better understanding of cross-reactivity of allergens.

The existing tools for allergenicity prediction can be divided into two groups. The first group is based on the Codex Alimentarius guidelines which state that a novel protein can be considered as a putative allergen if it shares greater than $35 \%$ sequence identity with a known allergen over a sliding window of at least 80 amino acids [37]. The tools in the second group use different bioinformatics approaches, statistical and machine learning techniques and widely varying validation procedures. In a recent study Maurer-Stroh et al. have made a comparison between the reported servers in recent literature results [38]. Authors compare the predictive performance of six methods: The Codex Alimentarius linear-window rule [37], AllerCatPro [38], PREAL [39], AllerHunter [40], AllergenFP [41] and AllerTOPv2 [42], using receiver operator characteristic parameters like specificity, sensitivity and accuracy. AllergenFP and AllerTOPv2 are developed in our Lab. The major weakness of this approach is the lack of conventional criteria for non-allergenic proteins [43]. To our knowledge, there is no existing server for cross-reactivity prediction.

In this study we have compiled a database of binders to the most common HLA alleles. The binders originate from known allergenic proteins of different species and routes of exposure. The peptides have been classified according to the HLA allele class II protein they bind. A comprehensive screening of the database reveals that different species share common HLA class II binders. Moreover, some of these binders are part of known linear IgE epitopes. The database and the searching algorithm have been implemented in a web application named AllerScreener, which is freely accessible at: http//www.ddg-pharmfac.net/AllerScreener. AllerScreener searches for potential binders to HLA class II alleles and predicts allergenicity and cross-reactivity among known and novel proteins.

\section{Databases and servers}

\subsection{Datasets}

A set of allergenic proteins has been collected from four online databases: Allergome [44], COMPARE (http://comparedatabase.org/), FARRP [45] and AllerBase [46]. Only proteins with known amino acid sequence have been chosen. Part of the protein sequences have been derived from UniProt database [47], as some of the databases do not contain information about the protein sequence of allergens.

Additionally, two sets of known linear B-cell epitopes and known T-cell epitopes have been derived from IEDB database [48].

\subsection{Servers for HLA binding prediction}

Each of the collected allergenic proteins has been screened for potential binders to HLA class II alleles by two in-house web servers: EpiTOP [49] and EpiDOCK [50]. 
In order to minimize the number of false positive binders, only peptides recognized as binders by both servers have been selected.

EpiTOP uses proteochemometrics models for HLA class II binding prediction. The binding affinity is predicted as $\mathrm{pIC}_{50}$ value. Nonamers with $\mathrm{pIC}_{50}>5.3$ $\left(\mathrm{IC}_{50}<5000 \mathrm{nM}\right)$ have been recognized as binders. EpiTOP predicts binding to 12 DRB1 alleles: DRB1*0101, DRB1*0301, DRB1*0401, DRB1*0404, DRB1*0405, DRB1*0701, DRB1*0802, DRB1*0901, DRB1*1101, DRB1*1201, DRB1*1302, DRB1*1501; 5 DQ alleles: DQA $1 * 0101 / \mathrm{DQB} 1 * 0501$, DQA1*0102/DQB1*0602, DQA1*0301/DQB1* 0302, DQA1*0401/DQB1*0402, DQA1*0501/DQB1*0301 and 7 DP alleles: DPA $1 * 0103 / \mathrm{DPB} 1 * 0201$, DPA1*0103/DPB $1 * 0401$, DPA $1 * 0103$ / DPB1*0402, DPA1*0201/DPB1*0101, DPA1*0201/DPB1*0501.

EpiDOCK prediction is based on quantitative matrices, derived by docking of combinatorial peptide libraries on HLA class II proteins. EpiDOCK predicts binding to the same alleles as EpiTOP and the additional DQA1*0501/DQB1*0201 allele.

\subsection{Web implementation}

The collected sets of binders to HLA alleles with their parent proteins and species, T-cell and B-cell epitopes have been compiled in a PostgreSQL database. The database is implemented on a web server using Django framework and Python.

\section{Results and discussion}

A dataset of known allergenic proteins has been collected from four databases: Allerbase - 1596 allergens, COMPARE - 2080 allergens, Allergome - 1704 allergens, and Allergen Online - 1975 allergens. Duplicates and sequences with unidentified residues have been removed. The final dataset contained 3961 allergenic proteins from different species and route of exposure.

A second dataset of peptide binders to the most common 25 HLA alleles has been compiled. The binders were generated by in silico prediction of the allergenic proteins. Each of the proteins was presented as a set of overlapping nonamer peptides. The affinities of the peptides to the HLA alleles have been predicted by EpiTOP and EpiDOCK. Only peptides predicted as binders by both servers were selected.

Additionally, two sets of 4875 known linear B-cell epitopes and 4963 known $\mathrm{T}$-cell epitopes were collected. They are used to identify known epitopes among the predicted HLA binders.

The datasets have been implemented on a web server for prediction of allergenicity and cross-reactivity of proteins. The server is named AllerScreener. AllerScreener predicts the presence of HLA class II binders in a tested protein and finds common binders with proteins from other species (cross-reactivity). If known $\mathrm{T}$-cell and/or B-cell epitopes are available among the predicted HLA binders, they are presented as well.

The workflow of AllerScreener is user-friendly and intuitive. There two options for prediction: Screening for HLA binders, T-cell and B-cell epitopes and Crossreactivity prediction. 
The Screening option consists of three steps (Fig. 1):

1. Enter the protein sequence in one letter code.

2. Select a HLA allele.

3. Start screening.

The result page returns a table containing:

1. Position of the binder in the query sequence. The query sequence could be a whole protein or a fragment of it.

2. Sequence of the binder.

3. UniProt ID of the parent protein.

4. Position of the binder in protein.

5. The species from which the protein originates.

6. Match in T-cell epitope dataset, if exists.

7. Match in B-cell epitope dataset, if exists.

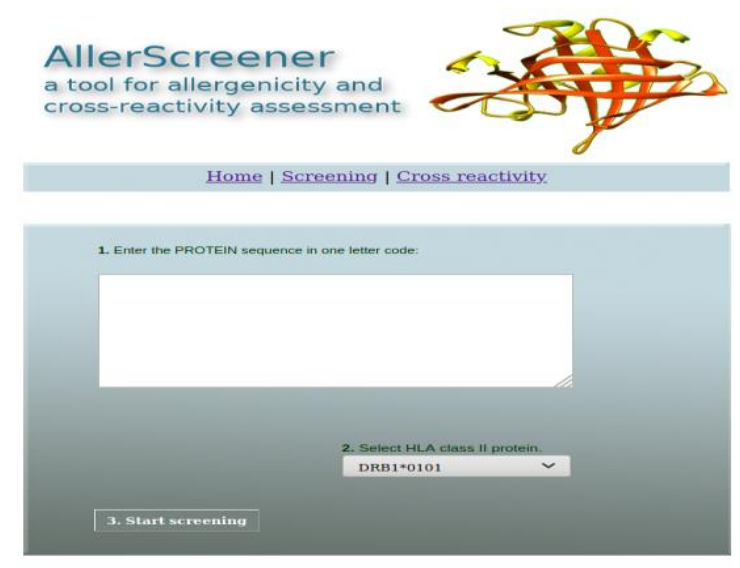

Fig. 1. AllerScreener front page

An example of the result page from the screening of alpha-s1-casein is given in Fig. 2. The result table lists the HLA binders in the query protein and the identical binders in other proteins from the same or different species. The common HLA binders underline the cross-reactivity between species. If the binder is present in any of the known T-cell or/and B-cell epitope, the probability for the parent protein to act as an allergen increases.

The cross-reactivity prediction also consists of three steps (Fig. 3):

1. Choose a species.

2. Chose a HLA allele.

3. Start screening.

AllerScreener returns a table containing:

1. A list of species with common predicted HLA binders.

2. The number of common HLA binders.

An example of a result page from the search for cross-reactivity of the species Acanthocheilonema viteae and HLA-DRB1*0101 allele is presented in Fig. 4. The higher number of common binders between the two species corresponds to higher probability of cross-reactivity between them. 


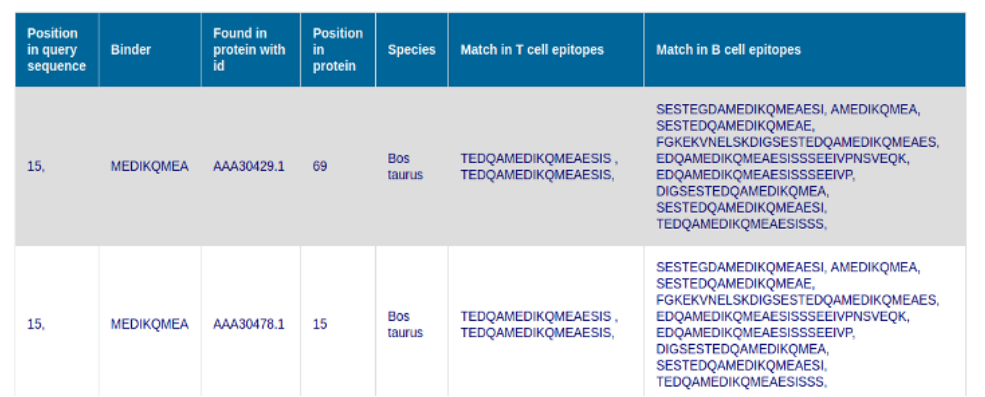

Fig. 2. AllerScreener result table for alpha-s1-casein and HLA-DRB1*0101 allele

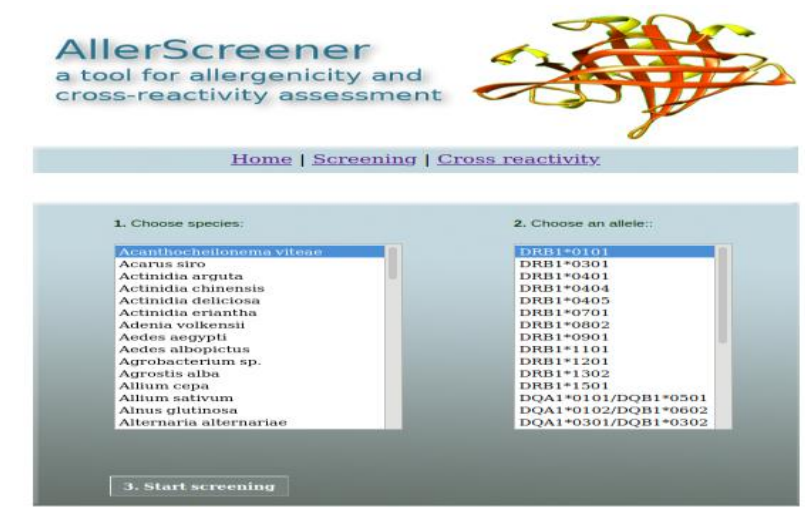

Fig. 3. AllerScreener cross-reactivity prediction front page

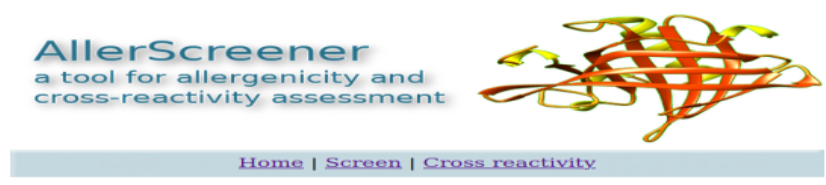

Export The Results To CSV File

Results for cross reactivity binders

Acanthocheilonema viteae DRB1*0101

\begin{tabular}{|l|l|}
\hline Species & Number of common binders \\
\hline Aedes aegypti & 5 \\
\hline Angiostrongylus vasorum & 9 \\
\hline Anisakis simplex & 12 \\
\hline Ascaris lumbricoides & 26 \\
\hline Ascaris suum & 62 \\
\hline Balanus rostratus & 1 \\
\hline Blattella germanica & 4 \\
\hline
\end{tabular}

Fig. 4. AllerScreener result table for cross-reactivity prediction of alpha-s1-casein and HLA-DRB $1 * 0101$ allele

The test set used by M a u r e r-S tr o h et al. [38] for the evaluation of six servers for allergenicity prediction has been applied to evaluate the performance of AllerScreener. It consists of 221 structurally non-redundant allergens and 221 likely 
non-allergens with the same as the allergens fold, derived from species like human, rice, yeast or Escherichia coli. AllerScreener predicts a protein from the test set as an allergen if there is at least one binder to an HLA allele. According to the study of Maurer-Stroh et al. AllerCatPro shows best performance with 100\% sensitivity, $67 \%$ specificity and $84 \%$ overall accuracy, followed by AllerTOPv2 (82\% sensitivity, $64 \%$ specificity and $73 \%$ overall accuracy) and AllergenFP (85\% sensitivity, 53\% specificity and $69 \%$ overall accuracy). AllerScreener recognized 218 of 221 allergens reaching $99 \%$ sensitivity. However, only 44 of 221 likely non-allergens have been recognized (20\% specificity). For 55 of the rest 177 false positives, AllerScreener found matches with known B-cell or T-cell epitopes. These matches cast doubt on the quality of the negative set used by M a u r e r-S troh et al. [38].

\section{Conclusion}

AllerScreener is a tool for allergenicity and cross-reactivity prediction based on the abilities of allergenic proteins to generate binders to HLA class II proteins. The peptides being generated are compared to HLA binders originating from known allergens. AllerScreener returns a list of common binders, origin proteins and species, as well as known T-cell and linear B-cell epitopes matching the binders.

Proteins of different species generate common HLA binders and determine the occurrence of cross-reactivity between them. AllerScreener allows searching for common HLA binders between species.

AllerScreener is user-friendly and freely accessible at:

http//www.ddg-pharmfac.net/AllerScreener/.

Acknowledgments: This work was accomplished by the financial support of Grant No BG05M2OP0011.001-0003, financed by the Science and Education for Smart Growth Operational Program (2014-2020) and co-financed by the European Union through the European structural and Investment funds.

\section{References}

1. Mc Conne11, T. H. The Nature of Disease: Pathology for the Health Professions. Baltimore, MD, Lippincott Williams \& Wilkins, 2007, p. 159.

2. Mak, T. W., M. E. S a unders, B. D. Jet t. Primer to the Immune Response. Second Edition. Immune Hypersensitivity. Academic Cell, 2014, pp. 487-516.

3. Ottenhoff, T. H., D. G. Elferink, J. Hermans, R. R. de Vries. HLA Class II Restriction Repertoire of Antigen-Specific T Cells. 1. The Main Restriction Determinants for Antigen Presentation are Associated with HLA-D/DR and Not with DP and DQ. - Hum Immunol, Vol. 13, 1985, pp. 105-116.

4. Jahn-Sch mid, B., W. F. Pi ckl, B. B ohle. Interaction of Allergens, Major Histocompatibility Complex Molecules, and T Cell Receptors: A Ménage à Trois That Opens New Avenues for Therapeutic Intervention in Type I Allergy. - International Archive Allergy and Immunology. Vol. 156. No 1, 2011, pp. 27-42. DOI:10.1159/000321904.

5. S ad an ag a, Y., et al. HLA Class II Association with Type I Allergy to House Dust Mite and Japanese Cedar Pollen in Japanese Subjects. - Allergology International, Vol. 47, Issue 4, pp. 285-291.

6. Morii, W., A. Sakai, T. Ninomiya, M. Kidoguchi, R. Sumazaki, S. Fujieda, E. Nogu chi. Association of Japanese Cedar Pollinosis and Sensitization with HLA-DPB1 in the Japanese Adolescent. - Allergology International, Vol. 67, 2018, Issue 1, pp. 61-66. 
7. W a ng, M., Z. M. Xing, D. L. Y u, Z. Y an, L. S. Y u. Association between HLA Class II Locus and the Susceptibility to Artemisia Pollen-Induced Allergic Rhinitis in Chinese Population. Otolaryngol Head Neck Surg., Vol. 130, 2004, No 2, pp. 192-196. DOI:10.1016/j.otohns.2003.08.012.

8. Jahn-Schmid, B., G. F. Fischer, B. Bohle, I. Faé, G. Gadermaier, A. Dedic, F. Ferre ir a, C. Ebne r. Antigen Presentation of the Immunodominant T-Cell Epitope of the Major Mugwort Pollen Allergen, Art V 1, is Associated with the Expression of HLA-DRB1*01. - Journal of Allergy and Clinical Immunology, Vol. 115, 2005, No 2, pp. 399-404. DOI: 10.1016/j.jaci.2004.10.010.

9. Torío, A., I. Sánchez-Guerrero, M. Muro et al. HLA Class II Genotypic Frequencies in Atopic Asthma: Association of DRB1*01-DQB1*0501 Genotype with Artemisia Vulgaris Allergic Asthma. - Hum. Immunol. Vol. 64, Aug 2003, No 8, pp. 811-815.

10. Cho, S. H., Y. K. Ki m, H. B. Oh, J. W. Jung, J. W. S on, M. H. Lee, H. S. Jee, Y. Y. Ki m, K. U. Min. Association of HLA-DRB1*07 and DRB1*04 to Citrus Red Mite (Panonychus Citri) and House Dust Mite Sensitive Asthma. - Clin. Exp. Allergy., Vol. 30, November 2000, No 11, pp. 1568-1575.

11. Lin, Y. C., C. C. Lu, H. J. S u, C. Y. Shen, H. Y. Lei, Y. L. Gu o. The Association between Tumor Necrosis Factor, HLA-DR Alleles, and IgE-Mediated Asthma in Taiwanese Adolescents. - Allergy., Vol. 57, September 2002, No 9, pp. 831-834.

12. Lara-Marquez, M. L., J. J. Yunis, Z. Layrisse, F. Ortega, E. Carvallo-Gil, S. Montagnani, N. J. Makhatadze, M. Pocino, C. Granja, E. Yunis. Immunogenetics of Atopic Asthma: Association of DRB1*1101 DQA1*0501 DQB1*0301 Haplotype with Dermatophagoides Spp.-Sensitive Asthma in a Sample of the Venezuelan Population. - Clin. Exp. Allergy., Vol. 29, January 1999, No 1, pp. 60-71.

13. Ivkovi c-Jurekovic, I., R. Zun ec, V. Balog, Z. Grubic. The Distribution of HLA Alleles among Children with Atopic Asthma in Croatia. - Coll. Antropol., Vol. 35, 2011, No 4, pp. 1243-1249.

14. La ma, M., M. Chatterje e, T. K. Chaudhuri. A Study of the Association of Childhood Asthma with HLA Alleles in the Population of Siliguri, West Bengal, India. - Tissue Antigens, Vol. 84, September 2014, No 3, pp. 316-20. DOI: 10.1111/tan.12403. Epub 25 Jun 2014.

15. Movahedi, M., M. Moin, M. Gharagozlou, A. Aghamohammadi, S. Dianat, B. Moradi, M. H. Nicknam, B. Nikbin, A. Amirzargar. Association of HLA Class II Alleles with Childhood Asthma and Total IgE Levels. - Iran Journal Allergy Asthma Immunol., Vol. 7, December 2008, No 4, pp. 215-220.

16. G a o, J., Y. Li n, C. Q i u, Y. Li u, Y. M a, Y. Li u. Association between HLA-DQA1. - DQB1 Gene Polymorphisms and Susceptibility to Asthma in Northern Chinese Subjects. - Chin. Med. Journal (Engl), Vol. 116, July 2003, No 7, pp. 1078-1082.

17. Gu o, X., P. Ni, L. Li. Association between Asthma and the Polymorphism of HLA-DQ Genes.Zhonghua Jie He He Hu Xi Za Zhi, Vol. 24, March 2001, No 3, pp. 139-141.

18. Chauhan, B., A. P. Knutsen, P. S. Hutcheson, R. G. Slavin, C. J. Bellone. T Cell Subsets, Epitope Mapping, and HLA-Restriction in Patients with Allergic Bronchopulmonary Aspergillosis. - Journal of Clinical Investigation, Vol. 97, 1996, pp. 2324-2331.

19. Chauhan, B., L. Santiago, D. A. Kirschmann, V. Hauptfeld, A. P. Knutsen, P. S. Hutcheson, S. L. Woulfe, R. G. Slavin, H. J. S chwartz, C. J. Bellon e. The Association of HLA-DR Alleles and T Cell Activation with Allergic Bronchopulmonary Aspergillosis. - Journal Immunol., Vol. 159, 1997, pp. 4072-4076.

20. Koehm, S., R. G. Slavin, P. S. Hutcheson, T. Trejo, C. S. David, C. J. Bellone. HLA-DRB1 Alleles Control Allergic Bronchopulmonary Aspergillosis-Like Pulmonary Responses in Humanized Transgenic Mice. - Journal of Allergy and Clinical Immunology, Vol. 120, September 2007, No 3, pp. 570-577. ISSN 0091-6749. DOI: 10.1016/j.jaci.2007.04.037.

21. Muro, M., P. Mondejar-López, M. R. Moya-Quiles et al. HLA-DRB1 and HLA-DQB1 Genes on Susceptibility to and Protection from Allergic Bronchopulmonary Aspergillosis in Patients with Cystic Fibrosis. - Microbiology Immunology, Vol. 57, March 2013, No 3, pp. 193-197. DOI: $10.1111 / 1348-0421.12020$. 
22. Howe11, W. M., S. J. Turner, J. O. Hourihane, T. P. De an, J. O. W arner. HLA Class II DRB1, DQB1 and DPB1 Genotypic Associations with Peanut Allergy: Evidence from Afamily-Based and Case-Control Study. - Clinical Experimental Allergy, Vol. 28, 1998, pp. 156-162.

23. Madore, A. M., V. T. Vaill an court, Y. A s a i et al. HLA-DQB1*02 and DQB1*06:03P are Associated with Peanut Allergy. - Eur. Journal Hum. Genet., Vol. 21, 2013, No 10, pp. 1181-1184. DOI:10.1038/ejhg.2013.13.

24. Dreskin, S. C., M. T. Tripputi, M. T. Aubrey, S. S. Mustafa, D. Atkins, H. L. Leo, B. Song, D. Schlichting, H. Talwar, Q. Wang, B. M. Freed. Peanut-Allergic Subjects and Their Peanut-Tolerant Siblings Have Large Differences in Peanut-Specific IgG That Are Independent of HLA Class II. - Clinical Immunology (Orlando, Florida), Vol. 137, December 2010, No 3, pp. 366-373. DOI: 10.1016/j.clim.2010.08.009.

25. Z h u, Z., P. H. L e e, M. D. $\mathrm{C} h$ a $f \mathrm{f} i \mathrm{n}, \mathrm{W} . \mathrm{C} \mathrm{h} \mathrm{u} \mathrm{g}, \mathrm{P}$. R. L o h, Q. L u, D. C. C h r i s t i a n i, L. L i a n g. A Genome-Wide Cross-Trait Analysis from UK Biobank Highlights the Shared Genetic Architecture of Asthma and Allergic Diseases. [Published Correction Appears in Nature Genetics, Vol. 50, December 2018, No 12, 1753]. Nature Genetics, Vol. 50, 2018, No 6, pp. 857-864. DOI:10.1038/s41588-018-0121-0.

26. Noguchi, E., H. Sakamoto, T. Hirota et al. Genome-Wide Association Study Identifies HLA-DP as a Susceptibility Gene for Pediatric Asthma in Asian Populations. - PlOS Genetics, Vol. 7, 2011, No 7, e1002170. DOI: 10.1371/journal.pgen.1002170.

27. Waage, J., M. Stand1, J. A. Curtin et al. Genome-Wide Association and HLA Fine-Mapping Studies Identify Risk Loci and Genetic Pathways Underlying Allergic Rhinitis [Published Correction Appears in Nat Genet., Vol. 50, September 2018, No 9, 1343]. - Nature Genetics, Vol. 50, 2018, No 8, pp. 1072-1080. DOI:10.1038/s41588-018-0157-1.

28. Ramasamy, Adaikalavan et al. A Genome-Wide Meta-Analysis of Genetic Variants Associated with Allergic Rhinitis and Grass Sensitization and Their Interaction with Birth Order. - Journal of Allergy and Clinical Immunology, Vol. 128, 2011, Issue 5, pp. 996-1005.

29. Pat e rn os te r, L., M. S t a n d, J. W a a g et al. Multi-Ancestry Genome-Wide Association Study of 21,000 Cases and 95,000 Controls Identifies New Risk Loci for Atopic Dermatitis. Nature Genetics, Vol. 47, 2015, No 12, pp. 1449-1456. DOI:10.1038/ng.3424.

30. Bønnelykke, K., M. C. Matheson, T. H. Pers et al. Meta-Analysis of Genome-Wide Association Studies Identifies Ten Loci Influencing Allergic Sensitization. - Nature Genetics, Vol. 45, 2013, No 8, pp. 902-906. DOI:10.1038/ng.2694.

31. Hong, X., K. Hao, C. Ladd-Acosta, et al. Genome-Wide Association Study identifies Peanut Allergy-Specific Loci and Evidence of Epigenetic Mediation in US Children. Nature Communications, Vol. 6, 2015, No 6304. https://doi.org/10.1038/ncomms7304

32. K h or, S. S., R. M or i n o, K. N a k a z o n o et al. Genome-Wide Association Study of Self-Reported Food Reactions in Japanese Identifies Shrimp and Peach Specific Loci in the HLA-DR/DQ Gene Region. - Scientific Reports, Vol. 8, 2018, No 1, 1069. Published 18 January 2018. DOI:10.1038/s41598-017-18241-w.

33. Krouse, J. H., M. J. Derebery, S. J. Chadwick. Principles of Allergy Management. Managing the Allergic Patient. New York, Saunders, 2008.

34. Canonica, G. W., I. J. An s ote gui, R. Pawankar et al. A WAO-ARIA-GALEN Consensus Document on Molecular-Based Allergy Diagnostics. - World Allergy Organ J., Vol. 6, 2013, No 17.

35. Westernberg, L., V. Schulten, J. A. Greenbaum et al. T-Cell Epitope Conservation across Allergen Species is a Major Determinant of Immunogenicity. - The Journal of Allergy and Clinical Immunology, Vol. 138, 2016, No 2, pp. 571-578.e7. https://doi.org/10.1016/j.jaci.2015.11.034

36. B u rast e r o, S. E. T-Cell Receptor-Mediated Cross-Reactivity to Different Allergens is Driven by Recognition of Homologous, Phylogenetically Conserved Epitopes. - Journal of Allergy and Clinical Immunology, Vol. 138, 2016, No 4, pp. 1237-1238.

37. CODEX Foods Derived from Modern Biotechnology. Second Ed. Food and Agriculture Organization of the United Nations, Codex Alimentarius Commission, Rome, 2009. 
38. Maurer-Stroh, S., N. Krutz, P. Kern, V. Gunalan, M. Nguyen, V. Limviphuvadh, F. Eis enhaber, F. Gerberick. AllerCatPro-Prediction of Protein Allergenicity Potential from the Protein Sequence. - Bioinformatics, Vol. 35, 1 September 2019, Issue 17, pp. 3020-3027. https://doi.org/10.1093/bioinformatics/btz029

39. Wang, J., D. Zhang, J. Li. PREAL: Prediction of Allergenic Protein by Maximum Relevance Minimum Redundancy (mRMR) Feature Selection. - BMC Syst. Biol., Vol. 7, 2013, Suppl. 5, S9. DOI: 10.1186/1752-0509-7-S5-S9.

40. Mu h, H. C., J. C. Tong, M. T. T a mmi. AllerHunter: A SVM-Pairwise System for Assessment of Allergenicity and Allergic Cross-Reactivity in Proteins. - PlOS One., Vol. 4, 2009, No 6, e5861. 10 June 2009. DOI:10.1371/journal.pone.0005861.

41. Dimitrov, I., L. Naneva, I. Doytchinova, I. Bangov. AllergenFP: Allergenicity Prediction by Descriptor Fingerprints. - Bioinformatics, Vol. 30, 15 March 2014, No 6, pp. 846-51. DOI: 10.1093/bioinformatics/btt619.

42. Dimitrov, I., I. B angov, D. R. Flower, I. Doytchinova. AllerTOP v.2 - A Server for In Silico Prediction of Allergens. - Journal Mol. Model, Vol. 20, June 2014, No 6, 2278. DOI: 10.1007/s00894-014-2278-5.

43. Remington, B., H. C. H. B roekman, W. M. B lom et al. Approaches to Assess IgE Mediated Allergy Risks (Sensitization and Cross-Reactivity) from New or Modified Dietary Proteins. Food Chem. Toxicol., Vol. 112, February 2018, pp. 97-107. DOI: 10.1016/j.fct.2017.12.025.

44. M ar i, A., C. R a s i, P. P a laz z o, E. S c a l a. Allergen Databases: Current Status and Perspectives. - Curr., Allergy Asthma Rep., Vol. 9, 2009, No 5, pp. 376-383. DOI:10.1007/s11882-009-0055-9.

45. Goodman, R. E., M. Ebis aw a, F. Ferreira et al. AllergenOnline: A Peer-Reviewed, Curated Allergen Database to Assess Novel Food Proteins for Potential Cross-Reactivity. - Mol. Nutr. Food Res., Vol. 60, 2016, No 5, pp. 1183-1198. DOI:10.1002/mnfr.201500769.

46. Ka d a m, K., R. Karbhal, V. K. Ja y ar a man, S. S a want, U. Kulkarni-Kale. AllerBase: A Comprehensive Allergen Knowledgebase. - Database, Vol. 2017, 2017, bax066. https://doi.org/10.1093/database/bax066

47. The UniProt Consortium. UniProt: the Universal Protein Knowledgebase. - Nucleic Acids Research, Vol. 46, 16 March 2018, Issue 5, p. 2699. https://doi.org/10.1093/nar/gky092

48. Zh an g, Q., P. W an g, Y. Ki m, P. H a s te-And er se n, J. B e a ver, P. E. B ourne et al. Immune Epitope Database Analysis Resource (IEDB-AR). - Nucleic Acids Res., Vol. 36, 2008, No W513-8. DOI:10.1093/nar/gkn254.

49. Dimitrov, I., P. Garnev, D. R. Flower, I. Doytchinova. EpiTOP - A Proteochemometric Tool for MHC Class II Binding Prediction. - Bioinformatics, Vol. 26, 2010, No 16, pp. 2066-2068. DOI:10.1093/bioinformatics/btq324.

50. Atan asova, M., I. Dimitrov, D. R. Flower, I. Doytchinova. EpiDOCK: A Molecular Docking-Based Tool for MHC Class II Binding Prediction. - Protein Eng. Des. Sel., Vol. 26, 2013, No 10, pp. 631-634.

Received: 15.09.2020; Second Version: 30.10.2020; Accepted: 4.11.2020 